\title{
Comparison of NEMA characterizations for Discovery MI and Discovery MI-DR TOF PET/CT systems at different sites and with other commercial PET/CT systems
}

\author{
Alexandre Chicheportiche ${ }^{*}$, Rami Marciano and Marina Orevi
}

\author{
* Correspondence: alexandre@ \\ hadassah.org.il \\ Department of Nuclear Medicine \\ and Biophysics, Hadassah Hebrew \\ University Medical Center, 91120 \\ Jerusalem, Israel
}

\begin{abstract}
Background: This article compares the physical performance of the 4-ring digital Discovery MI (DMI) and PMT-based Discovery MI-DR (DMI-DR) PET/CT systems. Physical performance was assessed according to the NEMA NU 2-2012 standards. Performance measures included spatial resolution, image quality, scatter fraction and count rate performance, and sensitivity. Energy and timing resolutions were also measured. Published DMI and DMI-DR performance studies from other centers are reviewed and compared.
\end{abstract}

Results: 4-ring DMI spatial resolution at 1-cm radial offset in the radial, tangential and axial directions was $4.62,4.18$ and $4.57 \mathrm{~mm}$, respectively, compared with the DMI-DR system values of $4.58,4.52$, and $5.31 \mathrm{~mm}$. Measured sensitivity was $13.3 \mathrm{kcps} /$ $\mathrm{MBq}$ at the center of the FOV and $13.4 \mathrm{kcps} / \mathrm{MBq} 10 \mathrm{~cm}$ off-center for the SiPMbased DMI system. DMI-DR system sensitivity was $6.3 \mathrm{kcps} / \mathrm{MBq}$ at the center of the FOV and $6.8 \mathrm{kcps} / \mathrm{MBq}$ at $10 \mathrm{~cm}$ off-center. DMI measured noise equivalent count rate peak was $175.6 \mathrm{kcps}$ at $20.1 \mathrm{kBq} / \mathrm{ml}$; DMI-DR was $146.7 \mathrm{kcps}$ at $31.7 \mathrm{kBq} / \mathrm{ml}$. Scatter fraction was $40.5 \%$ and $36.6 \%$, respectively. DMI image contrast recovery (CR) values ranged from 73.2\% (10 mm sphere) to $91.0 \%$ (37 mm sphere); DMI-DR, values ranged from $68.4 \%$ to $91.4 \%$. DMI background variability (BV) was 1.8\%-6.5\%; DMI-DR was $2.3 \%-9.1 \%$. The Q.Clear algorithm improved image quality, increasing $C R$ and decreasing BV in both systems. The photopeak energy resolution was $9.63 \%$ and $12.19 \%$ for DMI and DMI-DR, respectively. The time-of-flight (TOF) resolution was 377.26 ps and 552.71 ps, respectively. Compared with measurements in other centers, results were similar and showed an absolute mean relative deviation of $6 \%$ for DMl and $7 \%$ for DMI-DR overall performance results.

Conclusions: Performance measures were higher for the 4-ring DMI than the DMIDR system. The biggest advantages of the 4-ring DMI vs DMI-DR are improved sensitivity and count rate performance. This should allow a better image signal-tonoise ratio (SNR) for the same acquisition times or, similar SNR with lower acquisition times or injected activity. In its 3-ring configuration, the DMI showed worse performance results than the PMT-based system in terms of count rate scatter fraction and image quality (for similar axial FOV).

Keywords: NEMA, PET/CT, Physical performance, Discovery MI, Discovery MI-DR 


\section{Background}

Positron emission tomography/computed tomography (PET/CT) scanners have proven their role as multimodality medical imaging tools, and are now integrated into routine medical practice. General Electric (GE) Healthcare (Milwaukee WI, USA) recently launched the new digital Discovery MI (DMI) and PMT-based Discovery MI-Digital Ready (DMI-DR) whole body time-of-flight (TOF) PET systems. In an era when several different PET/CT scanners may be present in the same site or hospital, a thorough evaluation and comparison are important to optimize the use of all systems based on a deep knowledge of their similarities and differences.

National Electric Manufacturer's Association/Association of Electrical Equipment and Medical Imaging Manufacturers (NEMA) performance measurements are rigorous tests performed to ensure that imaging systems are fully operational and behave according to specifications. Measurements are performed before system acceptance and serve as a reference for future tests to make sure that the PET performances has not degraded over time. The standard NEMA NU 2-2012 guidelines for PET [1] includes a series of tests for spatial resolution, image quality, scatter fraction, and count rate performance, accuracy of correction for count losses and random events, and sensitivity.

The purposes of this work were to evaluate the physical performances of the SiPM-based 4-ring DMI and PMT-based DMI-DR PET/CT systems according to these standards and to compare their performance using the NEMA measurements. In addition, a comparison of NEMA data at our site with publicly available data for the 4-ring DMI system at Stanford and Uppsala Universities [2] and Tokyo [3] will be provided. A comparison with the 3-ring DMI system installed at Brugge [4] will be also presented. For the DMI-DR system, previous work from Southampton-Poole-Plymouth [5] will be considered for comparison. Finally, a comparison with NEMA measurements performed for other commercially available PET/ CT systems will be presented and discussed.

\section{Methods}

\section{PET/CT systems}

Discovery MI is the latest generation of PET/CT scanners commercialized by GE Healthcare and the first digital PET/CT system developed by the company. In early 2018, Hadassah-Hebrew University Medical Center received and installed the DMI PET/CT system. The GE DMI PET/CT system combines a 128-slice computed tomography (CT) system and a 4-ring PET system with LightBurst digital detectors providing a 20-cm axial field-of-view (AFOV) and a 70-cm transaxial field-of-view (TFOV). Each ring consists of 34 detectors, each of which contains four detector blocks, for a total of 544 detector blocks. Each detector block contains a $4 \times 9$ array of small lutetium-based scintillators (LBS), with crystals placed on three $3 \times 2$ arrays of silicon photomultiplier (SiPM) detectors, for a total of 19,584 crystals and 9792 SiPM channels. The SiPM signal readout electronics is implemented as an application-specific integrated circuit (ASIC). The output energy is digitized by an external analog-to-digital converter (ADC) and the timing signal by an external time-to-digital converter (TDC). Each crystal element is $3.95 \mathrm{~mm} \times 5.3 \mathrm{~mm}$ $\times 25 \mathrm{~mm}$, with several crystals connected to light guides that optimize light collection and improve sensitivity and resolution. The coincidence window is $4.9 \mathrm{~ns}$, and the lower (LLD) and upper (ULD) level energy discriminators are $425 \mathrm{keV}$ and $650 \mathrm{keV}$, respectively. 
These LBS crystals and SiPMs allow the DMI system to be TOF-capable with a timing resolution below $380 \mathrm{ps}$ [2]. Moreover, integrated real-time digital temperature sensors, coupled with a closed liquid cooling system, maintain SiPM detector temperature at $18^{\circ} \mathrm{C}$ to improve stability. Detector gain is adjusted in real-time as a function of temperature. The DMI system is also commercially available in a 3-ring configuration that provides a 15-cm AFOV.

A few months later, we also installed the GE DMI-DR system. The GE DMI-DR PET/CT system is a new PMT-based system with the capability to host the LightBurst Digital PET detectors combining the same 128-slice CT system. The DMI-DR AFOV and TFOV are equal to $15.6 \mathrm{~cm}$ and $70 \mathrm{~cm}$, respectively. In this model, each ring consists of 24 detectors. There are a total number of 13,824 LBS crystals for the whole system, with a $4.2 \mathrm{~mm} \times 6.3 \mathrm{~mm} \times 25 \mathrm{~mm}$ dimension of each element. Here also, several crystals are connected to light guides. The total number of photomultiplier tubes is 1024. The coincidence window and the LLD and ULD are the same as for the DMI system. The timing resolution for the Discovery MI-DR is close to $550 \mathrm{ps}$ [6]. Upgrade of DMI-DR to DMI involves replacement of the PET gantry (SiPMs instead of PMTs) but not of the CT gantry or stationary base. Additional requirements include adding non-gantry components such as a chiller, dehumidifier, power distribution unit, and a software update.

Both systems allow use of the same clinical reconstruction algorithms, namely VUE Point HD (VPHD), VUE Point FX (VPFX), and Q.Clear. VPHD is a 3D maximum likelihood ordered subset expectation maximization (3D OSEM) [7] image reconstruction algorithm that includes corrections for scatter, random events, dead time, and attenuation (from CT). VPFX is a VPHD algorithm that uses TOF information (3D OSEM + TOF) for better localization of the annihilation event, improving signal-to-noise ratio (SNR) in reconstructed images. VPHD and VPFX algorithms can also include point spread function (PSF) modeling to improve PET image contrast-to-noise ratio (CNR) [8], leading to their characterization as VPHD-S (3D OSEM + PSF) and VPFX-S (3D OSEM + TOF + PSF) reconstruction algorithms, respectively. The most recent Q.Clear algorithm is a Bayesian penalized-likelihood iterative image reconstruction algorithm $[9,10]$, where the sole user-input value, termed $\beta$, controls the relative strength of the noise regularizing term. A typical $\beta$ value for ${ }^{18}$ F-FDG examinations is around 400 [10]. The advantage of such an algorithm, in contrast with OSEM, is that Q.Clear reaches full convergence [11] without the effects of excessive noise with increasing iterations, thereby maintaining good image quality.

\section{NEMA performance measurements}

\section{Spatial resolution}

Spatial resolution measurements were performed according to the NEMA NU-2 2012 procedure [1] using a set of ${ }^{18} \mathrm{~F}$ point sources with a diameter of less than $1 \mathrm{~mm}$ radially and axially. The activity per source was about $1.5 \mathrm{MBq}$ for the two systems. Point sources were inserted inside 75-mm-long glass capillary tubes (BRIS Micro-Hematocrit Capillary Tube, Thomas Scientific, Swedesboro, NJ, USA; soda-lime glass, $1.15 \mathrm{~mm}$ inner and $1.5 \mathrm{~mm}$ outer diameter, respectively). Measurements were performed with sources placed at 1,10 , and $20 \mathrm{~cm}$ radial offset vertically, and at axial positions $\mathrm{z}_{0}=0 \mathrm{~cm}(\mathrm{CFOV})$ 
and $\mathrm{z}_{1}=3 / 8 \times$ AFOV offset from the center of the axial FOV $\left(\mathrm{z}_{1}=7.5 \mathrm{~cm}\right.$ for Discovery $\mathrm{MI}$ and $\mathrm{z}_{1}=5.9 \mathrm{~cm}$ for Discovery MI-DR). Data were collected for $1 \mathrm{~min}$ at $\mathrm{z}_{0}$ and for 4 $\min$ at $z_{1}$ to ensure similar counts in the two axial positions; at least $2 \times 106$ counts at each position. Acquired data were first reconstructed using the filter back projection (FBP) algorithm into a $384 \times 384 \times 71$ matrix, without any filtering, as specified in the NEMA procedure [1]. Additional reconstructions were performed using the VPHD (3D OSEM) and VPHD-S (3D OSEM + PSF) algorithms in a $384 \times 384 \times 71$ matrix with 4 iterations, 34 (DMI)/24 (DMI-DR) subsets, and a 2.0-mm Gaussian filter. Reconstruction parameters (matrix, number of iterations, subsets and filter value) were chosen according to "ideal" parameters specified in the "NEMA Test Procedures and Detector Performance test" brochures for DMI [12] and DMI-DR [13] provided by the vendor. Radial, tangential, and axial spatial resolutions at 1,10 , and $20 \mathrm{~cm}$ radial offset were averaged over the two axial positions and presented as the full width half maximum (FWHM) and the full width tenth maximum (FWTM) of the reconstructed PSF. Results were obtained using vendor-integrated software.

\section{Image quality}

The NEMA image quality test was established to provide an overall assessment of the imaging capabilities of the system under conditions resembling clinical scans. This test uses the NEMA IEC image quality body phantom (IQBP) (Model PET/IEC-BODY/P) [1], which contains spheres with an internal diameter of 10,13,17,22, 28, and $37 \mathrm{~mm}$ and a 50-mm diameter cylindrical insert mounted in the center. The 10, 13, 17, and 22 $\mathrm{mm}$ spheres were filled with radioactive material $\left({ }^{18} \mathrm{~F}\right)$, while the two largest spheres held nonradioactive water. The lung insert provided with the phantom was filled with low-density material (polystyrene) and water. The 70-cm-long NEMA scatter phantom (Model PET/NEMA-SCT/P), used for count rate and scatter fraction performance measurements, was also used in this test. A line source inserted through the body of the scatter phantom was filled with a solution of radioactive ${ }^{18} \mathrm{~F}$ and water. The scatter phantom was placed adjacent to the IQBP and served as a source of out-of-field background activity in order to simulate clinical imaging conditions.

For the DMI system evaluation, the line source of the scatter phantom was filled with 99.2 $\mathrm{MBq}(2.7 \mathrm{mCi})$ of ${ }^{18} \mathrm{~F}$, and the IQBP background region and spheres contained an ${ }^{18} \mathrm{~F}$ activity concentration of $6.37 \mathrm{kBq} / \mathrm{mL}(0.17 \mu \mathrm{Ci} / \mathrm{mL})$ and $25.48 \mathrm{kBq} / \mathrm{mL}(0.69 \mu \mathrm{Ci} /$ $\mathrm{mL})$, respectively, at the time of the first acquisition.

For the DMI-DR evaluation, the phantom background region and spheres contained $5.6 \mathrm{kBq} / \mathrm{mL}(0.15 \mu \mathrm{Ci} / \mathrm{mL})$ and $22.4 \mathrm{kBq} / \mathrm{mL}(0.61 \mu \mathrm{Ci} / \mathrm{mL})$, respectively, at the time of the first acquisition. The line source contained $111.7 \mathrm{MBq}(3.0 \mathrm{mCi})$ of ${ }^{18} \mathrm{~F}$ at that time.

In both cases, the IQBP was filled to reach a target-to-background ratio of 4:1. Three decay-adjusted acquisitions of 271, 279, and $282 \mathrm{~s}$ on DMI, and 212, 217, and $222 \mathrm{~s}$ on DMI-DR were performed, and data were corrected for random coincidences, normalization, dead-time losses, and scatter. A CT transmission scan (140 kV, auto mA) was acquired before the PET emission scans for attenuation correction. Data were reconstructed with the clinical VPHD (3D OSEM), VPHD-S (3D OSEM + PSF), VPFX (3D OSEM + TOF), and VPFX-S (3D OSEM + TOF + PSF) reconstruction algorithms in a $384 \times 384 \times 71$ matrix, with 4 iterations, $34(\mathrm{DMI}) / 24$ (DMI-DR) subsets, and a $2-\mathrm{mm}$ 
Gaussian filter. Here also, reconstruction parameters were chosen accordingly to [12, 13]. Q.Clear reconstructions with $\beta=50,200$, and 350 were also performed for comparison. A value of $\beta=350$ corresponds to the value used in clinical studies, as recommended by the manufacturer.

Contrast recovery (CR) and background variability (BV) were quantified using the integrated vendor software. The average and standard deviation (SD) of CR and BV were calculated over the three acquisition scans.

\section{Count rate performance and scatter fraction}

To determine the count rate response of the detectors at different activity levels in the PET FOV and the number of scattered-to-total events, the cylindrical $70-\mathrm{cm}$-long scatter phantom was used according to NEMA procedure [1]. The phantom was positioned such that its center was aligned with the center of the FOV. Following vendor recommendations, a line source was filled with $819 \mathrm{MBq}(22.1 \mathrm{mCi})$ of ${ }^{18} \mathrm{~F}$ for the DMI evaluation and with $1054 \mathrm{MBq}(28.5 \mathrm{mCi})$ for the DMI-DR evaluation at the start of the acquisitions so that the peak count rate of the systems would be exceeded. The source was placed inside the phantom with a $4.5-\mathrm{cm}$ vertical offset. Data were acquired for about $10 \mathrm{~h}$ and over a total of 24 frames (17 frames of $15 \mathrm{~min}$ followed by 7 frames of 25 min with a 25-min delay between consecutive frames). Prompts, noise equivalent count rate (NECR), trues, random and scatter events, and the scatter fraction were calculated for both PET/CT systems using the vendor-integrated software.

\section{Accuracy of correction for count losses and random events}

Data acquired for the count rate performance and scatter fraction tests reconstructed using the VPHD (3D OSEM) algorithm in a $128 \times 128$ matrix were used for this test. Count loss and random correction accuracy were calculated below the peak NEC by comparing the trues rates (i) calculated using count losses and random corrections, and (ii) the true rates extrapolated from measurements (least-square fit) with negligible count losses and random events. Mean, minimum, and maximum deviations were obtained using the vendor-integrated software and recorded for each system.

\section{Sensitivity}

The sensitivity test measures the coincidence detection rate of the system for a given amount of activity in the FOV. In accordance with NEMA specifications [1], sensitivity was measured using a 70-cm-long thin line filled with an amount of ${ }^{18} \mathrm{~F}$ activity low enough to minimize any dead time in the system: $2.51 \mathrm{MBq}(68 \mu \mathrm{Ci})$ for DMI and 2.80 $\mathrm{MBq}(76 \mu \mathrm{Ci})$ for DMI-DR at the start of the acquisitions. In order to ensure that all positrons annihilate near the decay site, the line source was placed inside high-density material that stops all positrons. NEMA attenuation-free sensitivity in the air was obtained from successive $1 \mathrm{~min}$ measurements of the count rate using five aluminum sleeves of known thicknesses (phantom Model PET/NEMA-SEN/P). The data were exponentially extrapolated to zero sleeve thickness, as described first by Bailey et al. [14]. Measurements were performed at the center of the FOV and at a 10-cm horizontal offset. Data were analyzed using the vendor-integrated software after random subtraction to ensure true-only datasets. NEMA attenuation-free sensitivity in the air and the axial 
sensitivity profile at the center of the FOV and at a 10 -cm radial offset were obtained with the vendor-integrated software.

All ${ }^{18} \mathrm{~F}$ activity measurements for phantom preparations were done using a Capintec $\mathrm{CRC}^{\circ}-55 \mathrm{tR}$ dose calibrator, channel \#472 (calibrated with respect to national standards).

\section{Energy and coincidence timing resolution}

In addition, since the DMI and DMI-DR are TOF PET/CT systems, TOF performance was evaluated using another methodology. This last test also measured energy resolution for these two systems.

The timing resolution and energy resolution were evaluated using an experimental setup similar to the one in [15]. The 70-cm-long line source used during the sensitivity test was filled with $19.23 \mathrm{MBq}(0.52 \mathrm{mCi})$ of ${ }^{18} \mathrm{~F}$ for DMI and $15.51 \mathrm{MBq}(0.42 \mathrm{mCi})$ for DMI-DR. The line source was placed inside a single aluminum sleeve at the CFOV in the axial direction. A 30-min acquisition was performed, yielding a minimum of 300 million counts. Calculation of the timing resolution FWHM was based on the Coincidence Time Correction (CTC) procedure described in [16] by fitting the timing spectra peaks for each crystal pair (for each line of response). The energy resolution was computed for each crystal and at each block level for all the modules. The system energy/ timing resolution were quantified as the average of FWHM energy/timing values for each block.

\section{Comparisons with other studies}

The NEMA NU 2-2012 parameters measured for the 4-ring DMI system (AFOV $=20$ $\mathrm{cm}$ ) were compared with results obtained at Stanford and Uppsala Universities [2] and Tokyo [3] for the same system and with results from Brugge [4] obtained for the system in its 3-ring configuration (AFOV $=15 \mathrm{~cm}$ ). For the DMI-DR system, results were compared with those obtained at Southampton-Poole-Plymouth [5].

In a second step, a comparison was made between results obtained in our study and those published for other commercially available systems from Siemens Healthcare, Erlangen, Germany (Biograph mCT Flow [17, 18], Biograph mMR PET/MR [18, 19] and digital Biograph Vision [20]), Philips Medical Systems, Eindhoven, The Netherlands (Ingenuity TF [21] and Vereos [22, 23]) and GE Healthcare, Milwaukee, WI, USA (Discovery IQ [24, 25] and SiPM-based Signa PET/MR [26]).

\section{Results}

System performance

\section{Spatial resolution}

The radial, tangential, and axial spatial resolution, averaged over the two axial positions $z_{0}$ and $z_{1}$, for a radial offset of 1,10 , and $20 \mathrm{~cm}$ of the point source in terms of FWHM and FWTM for DMI and DMI-DR are listed in Table 1. Data are presented for the FBP and the clinical 3D-OSEM reconstruction algorithms, with and without modeling of the PSF. It is noteworthy that the results for the two systems are similar with, for instance, a spatial resolution for DMI and DMI-DR of 4.62 vs 4.58 for FBP, 3.85 vs 4.13 for VPHD (3D OSEM) and 2.70 vs 2.84 for VPHD-S (3D OSEM + PSF) at 1-cm radial offset. 


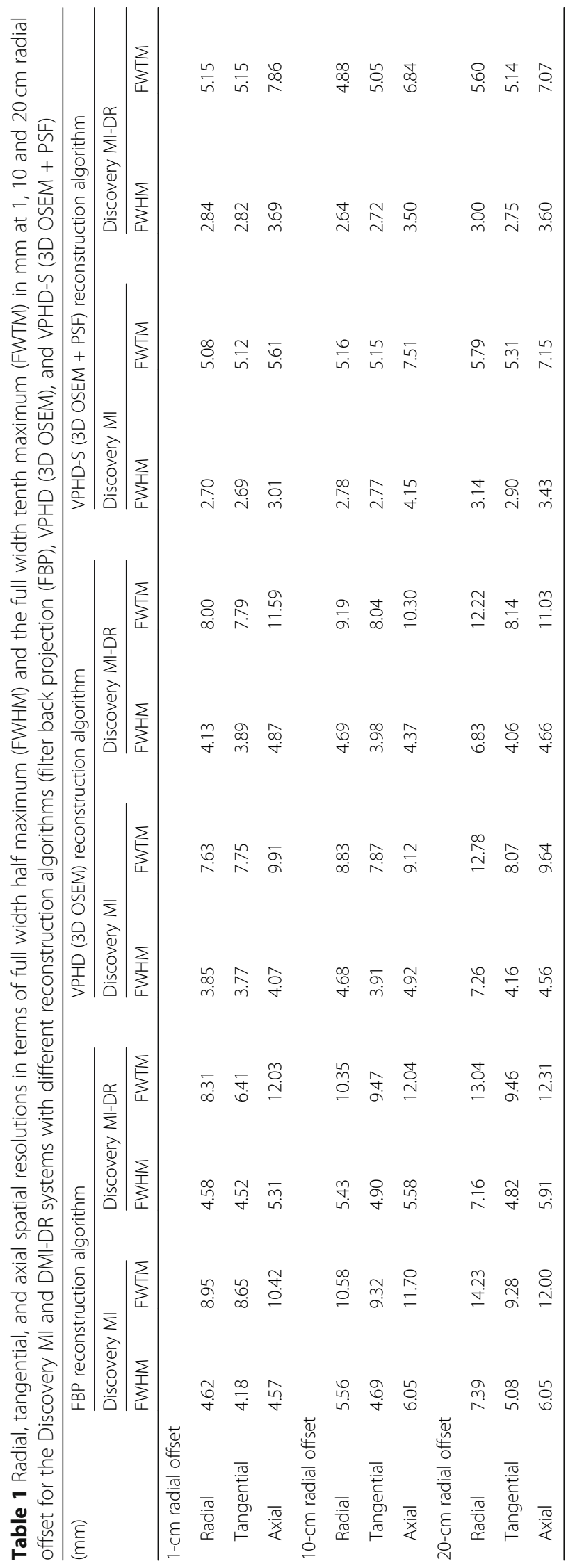


For the DMI evaluation, the results obtained at our institution are compared with previous results from Stanford and Uppsala Universities [2], Tokyo [3] and Brugge [4] in Fig. 1a-c. The latter show that the spatial resolution from the DMI system across the five centers is very similar, regardless of the 3-ring or 4-ring configuration.

For DMI-DR, Fig. 1d-f also shows comparable results across the different centers.

\section{Image quality}

CR and BV obtained from images acquired using the NEMA NU 2-2012 IQBP phantom and reconstructed using the VPHD (3D OSEM), VPHD-S (3D OSEM + PSF), VPFX (3D OSEM + TOF), VPFX-S (3D OSEM + TOF + PSF) and Q.Clear $(\beta=50,200,350)$ algorithms are shown in Fig. 2a and b for DMI and DMI-DR systems. For all reconstruction algorithms, better CR and BV results were obtained for DMI compared with DMI-DR, with

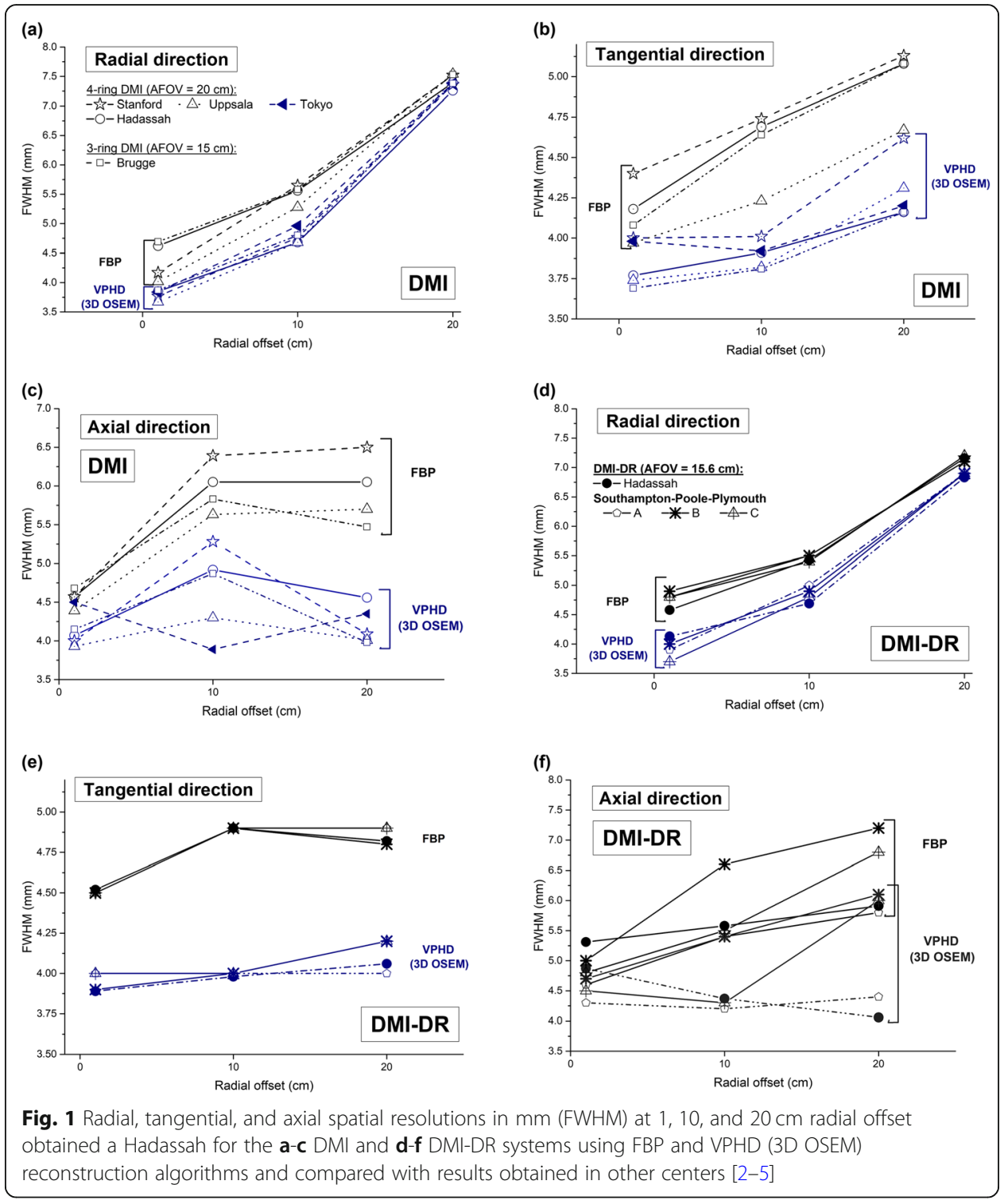




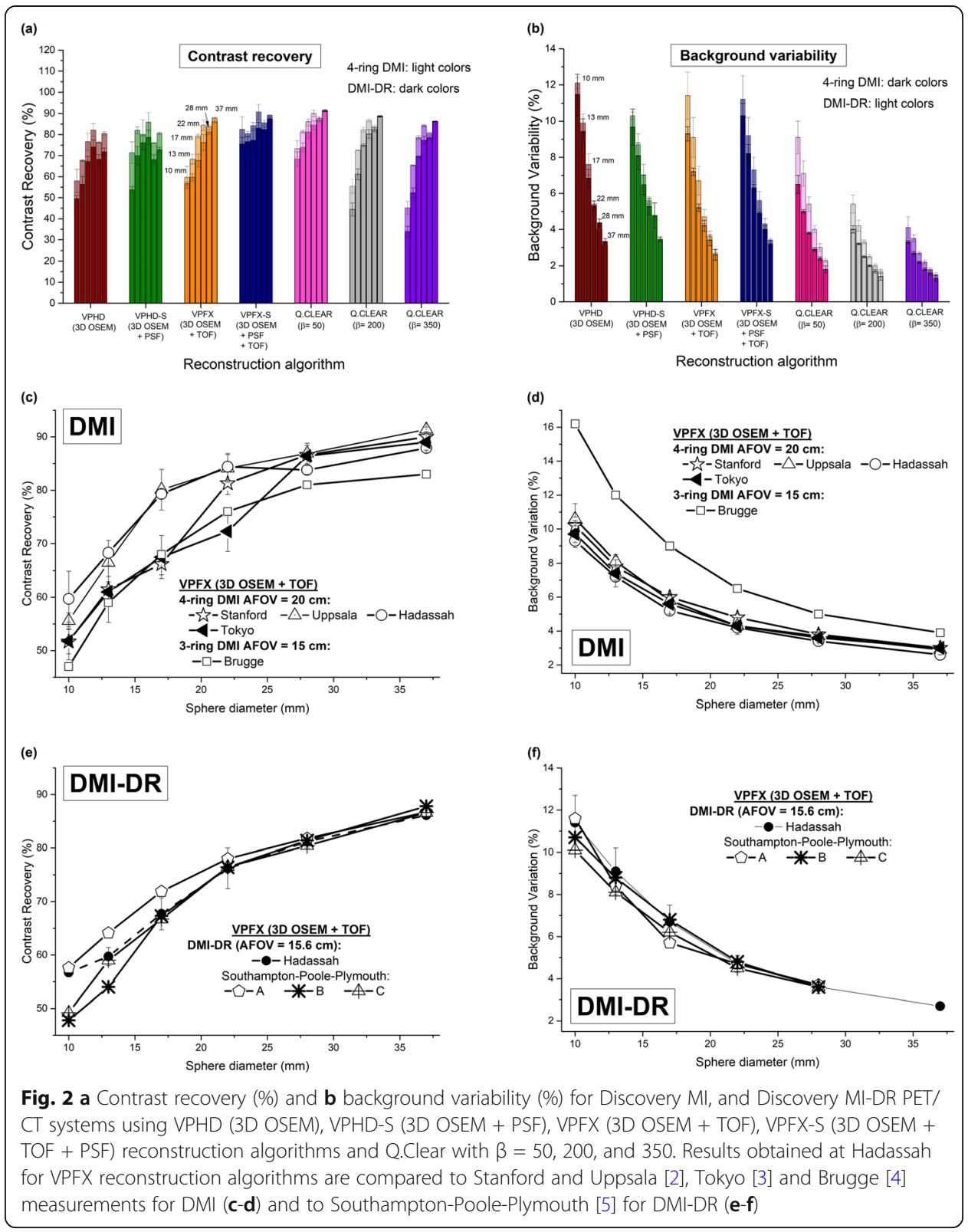

a mean minimum/maximum relative difference of $4.6 \%(\mathrm{QC} \beta=50) / 12.5 \%$ (VPHD) for $\mathrm{CR}$, and $1.5 \%(\mathrm{VPHD}) / 35.8 \%(\mathrm{QC} \beta=50)$ for BV.

For both systems, the use of PSF increased hot sphere, but not cold sphere contrast. The addition of the TOF information to PSF resulted in an overall improvement in CR and BV. The Q.Clear algorithm improved the image quality by further decreasing the BV. Indeed, the VPFX-S and QC $(\beta=50)$ reconstruction algorithms presented a similar CR (slightly higher for QC), but a lower BV for QC. It is noteworthy that CR and BV decreased with increasing values of the $\beta$ parameter although all $Q C$ results remained similar.

Figure 2c, d presents the results obtained for the DMI system at Stanford and Uppsala Universities [2], Tokyo [3] and Brugge [4] in comparison with the results obtained at our institution using the VPFX algorithm. It is noteworthy that image quality results are very similar across centers, apart from the BV obtained at Brugge for the 
3-ring system, with a minimum/maximum relative deviation of $74 \% / 50 \%$ from our results is obtained.

Figure 2e, f shows similar image quality results obtained at Southampton-PoolePlymouth [5] and our center for the DMI-DR system.

Figure 3 presents the transverse views of the phantom acquisitions for the VPDH, VPFX, VPHD-S, VPFX-S, and Q.Clear $(\beta=50,350)$ algorithms. A visual appreciation of the phantom acquisitions confirms the previous observations. The images illustrate better quality when using TOF information for the two PET/CT systems.

\section{Count rate performance and the scatter fraction}

Figure $4 \mathrm{a}$ and $\mathrm{b}$ shows, respectively, the count rate performance and the scatter fraction of the DMI and DMI-DR systems. The peak NECR is $175.6 \mathrm{kcps}$ at $20.1 \mathrm{kBq}$ for DMI while, for DMI-DR, its value reaches $146.7 \mathrm{kcps}$ at $31.7 \mathrm{kBq}$. At clinical activity concentrations, i.e., at about $5 \mathrm{kBq} / \mathrm{mL}$, the NECR is $95.3 \mathrm{kcps}$ for DMI and $58.5 \mathrm{kcps}$ for DMI-DR. The scatter fraction of the two systems was slightly different over the range of activity concentrations, with values of $40.5 \%$ vs $36.5 \%$ at peak NECR, and $38.7 \%$ vs $34.3 \%$ at $5 \mathrm{kBq} / \mathrm{mL}$, for the DMI and DMI-DR systems, respectively.

Figure 4c compares the DMI scatter fraction to results from Stanford and Uppsala Universities [2], Tokyo [3] and Brugge [4]. Table 2 summarizes the count rate and scatter fraction results obtained for the two systems at different sites.

\section{Accuracy of the corrections for count losses and random events}

The mean error for count rate accuracy was similar for DMI (3.11\%) and DMI-DR (3.23\%) at peak NECRs. For activity concentration in the clinical range, the mean error was $0.6 \%$ for DMI and $1.0 \%$ for DMI-DR.

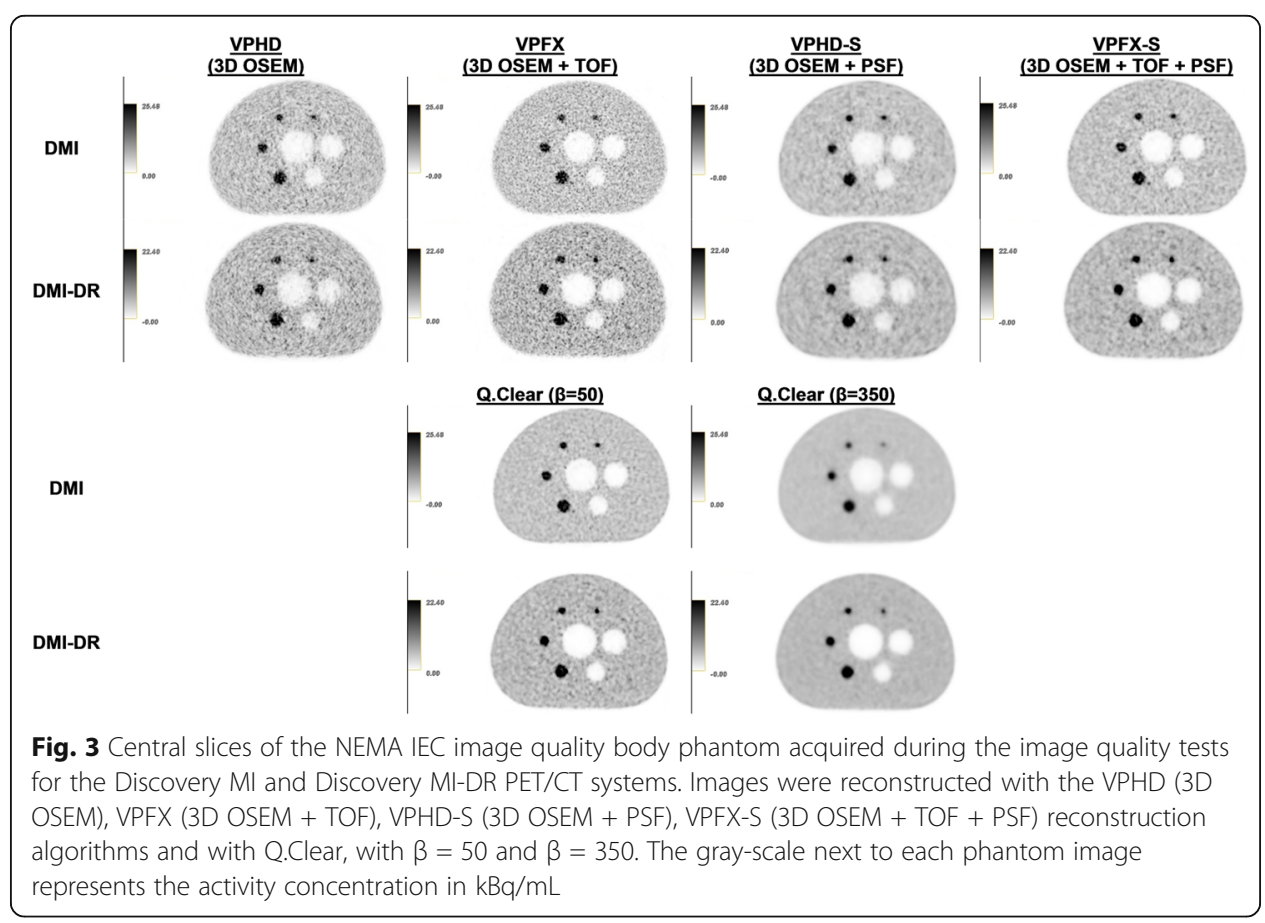



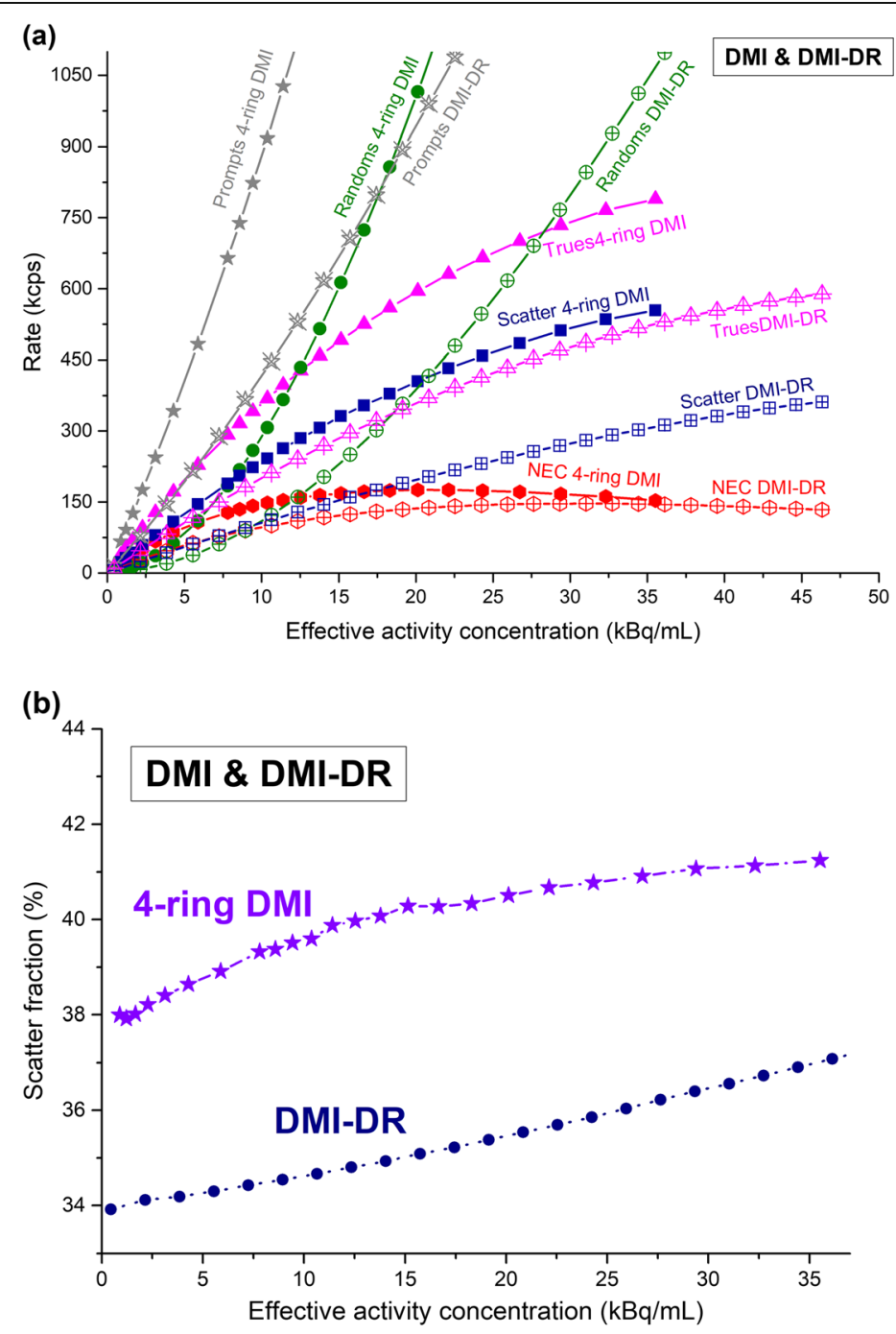

(c)

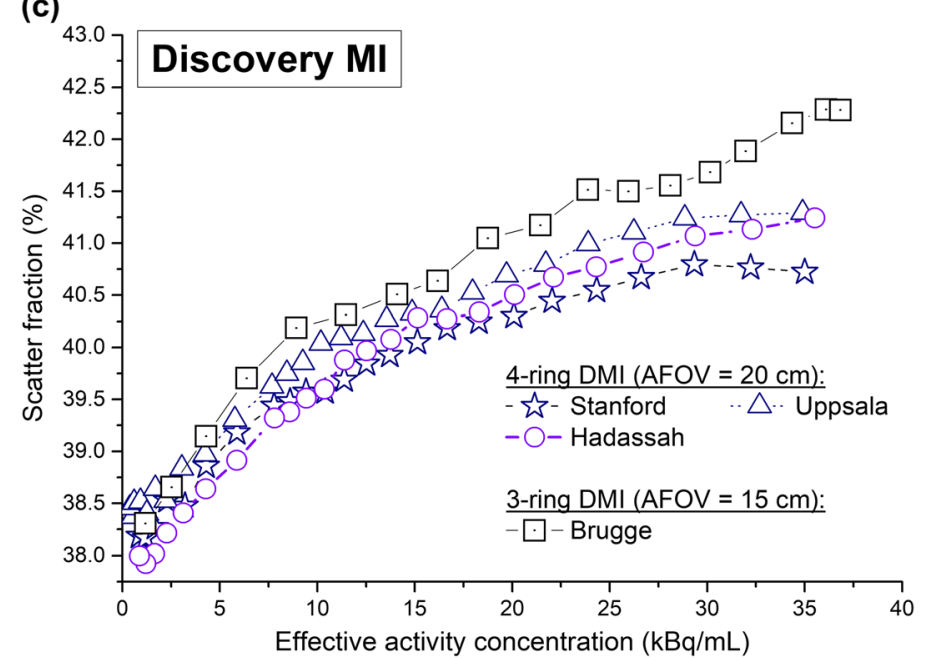

Fig. 4 (See legend on next page.) 
(See figure on previous page.)

Fig. 4 Count rate performance (prompts, NECR, true, random and scatter values) in kcps (a) and scatter fraction (b), versus the effective activity concentration in $\mathrm{kBq} / \mathrm{mL}$ for the Discovery Ml and Discovery MI-DR PET/CT systems at Hadassah. Comparison of the scatter fraction obtained at Hadassah, Stanford, and Uppsala [2] and Brugge [4] for the DMI system is presented in (c)

\section{Sensitivity}

The sensitivity at CFOV for the digital DMI system was $13.3 \mathrm{cps} / \mathrm{kBq}$ and 13.4 $\mathrm{cps} / \mathrm{kBq}$ at $10-\mathrm{cm}$ radial offset. For the DMI-DR system, the sensitivity was 6.3 $\mathrm{cps} / \mathrm{kBq}$ and $6.8 \mathrm{cps} / \mathrm{kBq}$, respectively. Figure 5 shows (a) the slice sensitivity profile at CFOV and (b) the sensitivity at CFOV and at $10-\mathrm{cm}$ radial offset for five aluminum sleeve thickness with the extrapolation of the NEMA attenuation-free sensitivity in the air (without attenuation material) for the 4-ring DMI and DMIDR systems.

The results obtained at Uppsala and Stanford Universities [2] and Tokyo [3] for the 4ring DMI system were $13.4 \mathrm{cps} / \mathrm{kBq}, 14.0 \mathrm{cps} / \mathrm{kBq}$ and $12.6 \mathrm{cps} / \mathrm{kBq}$ at CFOV, respectively. For the 3-ring DMI system, a sensitivity at CFOV of $7.3 \mathrm{cps} / \mathrm{kBq}$ was obtained at Brugge [4].

For the DMI-DR system, sensitivities at CFOV of $7.6 \mathrm{cps} / \mathrm{kBq}, 6.8 \mathrm{cps} / \mathrm{kBq}$ and 7.4 $\mathrm{cps} / \mathrm{kBq}$ were obtained at Southampton-Poole-Plymouth [5].

Figure $5 c$ and d shows the NEMA sensitivity results obtained in the different centers for DMI and DMI-DR.

\section{Energy and coincidence timing resolution}

The system average energy resolution was $9.63 \% \pm 0.08 \%$ for DMI and $12.19 \% \pm 0.09 \%$ for DMI-DR in terms of FWHM. The system average timing resolution was $377.26 \pm$ 2.62 ps for DMI and $552.71 \pm 6.67$ ps for DMI-DR.

At Stanford and Uppsala [2], photopeak energy resolution was $9.44 \pm 0.07 \%$ and $9.35 \% \pm 0.05 \%$ and the timing resolution was $374.16 \pm 2.60 \mathrm{ps}$ and $376.76 \pm 2.70 \mathrm{ps}$, respectively. At Brugge [4] energy resolution was $9.30 \pm 0.06 \%$ and the timing resolution was $375.60 \pm 2.70$ ps.

Table 2 Peak noise equivalent count rate (NECR), scatter fraction, peak true count rate and their corresponding activity concentration for the DMI-DR and DMI systems. Results obtained for DMIDR at Southampton, Poole, and Plymouth (scanners A, B, C) [5] and for DMI at Stanford and Uppsala Universities [2], Tokyo [3] and Brugge [4] are listed for comparison

\begin{tabular}{|c|c|c|c|c|c|c|c|c|c|}
\hline \multirow[t]{3}{*}{ Measurement } & \multicolumn{4}{|l|}{ MI-DR } & \multicolumn{5}{|l|}{$\mathrm{Ml}$} \\
\hline & \multicolumn{4}{|c|}{$\mathrm{AFOV}=15.6 \mathrm{~cm}$} & \multicolumn{4}{|l|}{$\mathrm{AFOV}=20 \mathrm{~cm}$} & \multirow{2}{*}{$\begin{array}{l}\text { AFOV }=15 \mathrm{~cm} \\
\text { Brugge }\end{array}$} \\
\hline & Present work & $A$ & B & $C$ & Present work & Stanford & Uppsala & $\overline{\text { Tokyo }}$ & \\
\hline Peak NECR (kcps) & 146.7 & 144 & 139 & 142 & 175.6 & 201.1 & 185.7 & 185.6 & 102.3 \\
\hline $\begin{array}{l}\text { Activity at peak } \\
\operatorname{NECR}(\mathrm{kBq} / \mathrm{mL})\end{array}$ & 31.7 & 27.3 & 30.7 & 27.7 & 20.1 & 22.1 & 21.7 & 22.5 & 23.0 \\
\hline $\begin{array}{l}\text { Scatter fraction at } \\
\text { peak NECR (\%) }\end{array}$ & 36.6 & 36.8 & 37.5 & 36.9 & 40.5 & 40.4 & 40.8 & 42.1 & 41.2 \\
\hline $\begin{array}{l}\text { Peak true count } \\
\text { rate (kcps) }\end{array}$ & 589.1 & & & & 789.2 & 875.9 & 827.0 & & 463.1 \\
\hline $\begin{array}{l}\text { Activity at peak true } \\
\text { count rate }(\mathrm{kBq} / \mathrm{mL})\end{array}$ & 46.3 & & & & 35.5 & 35.4 & 34.8 & & 36.9 \\
\hline
\end{tabular}



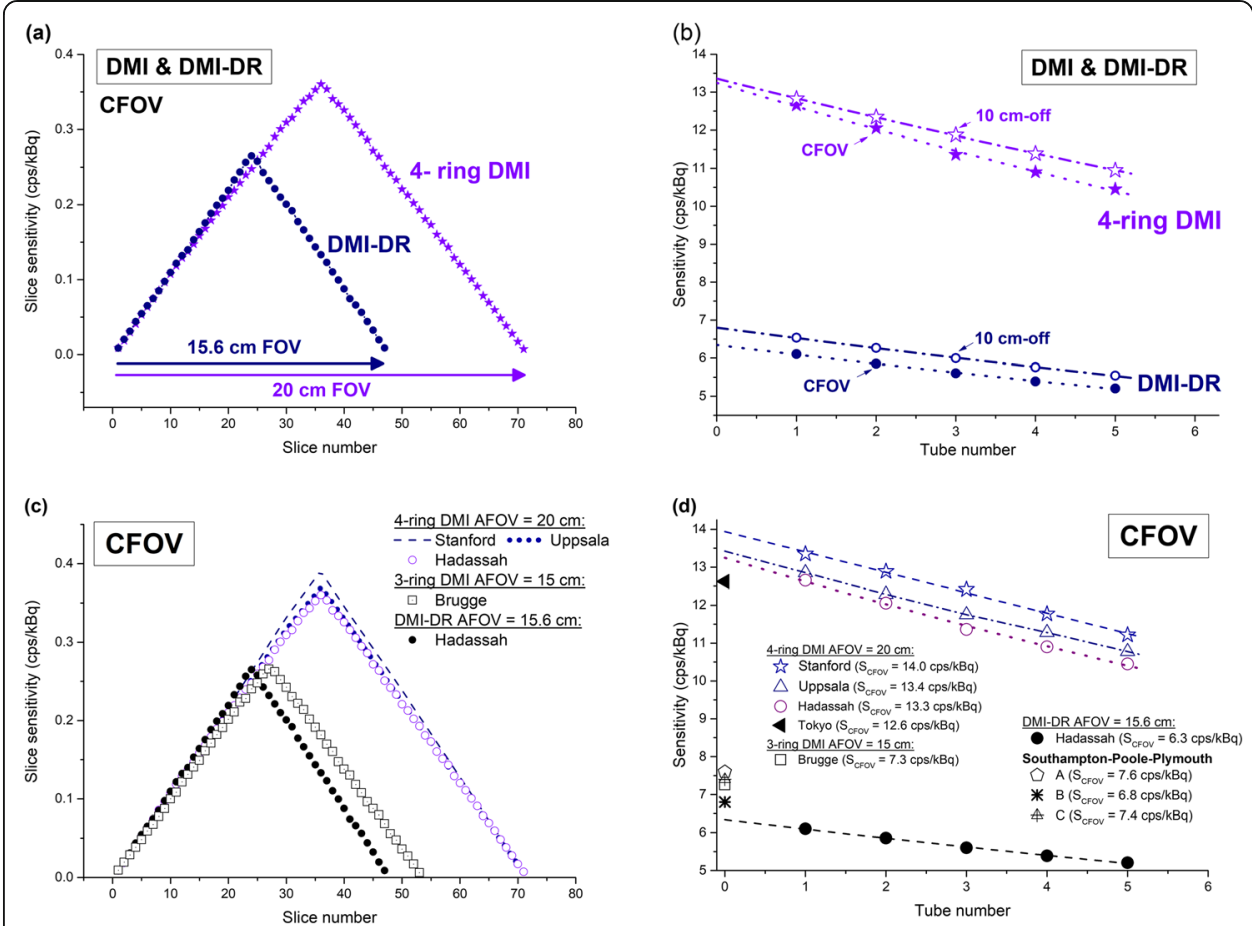

Fig. 5 a Slice sensitivity profile at CFOV for DMI and DMI-DR. b Successive measurements of the count rate at CFOV and 10-cm radial offset using five aluminum sleeves and an extrapolation process to get the NEMA attenuation-free sensitivity in air. Comparison of the sensitivity results for the DMI and DMI-DR systems with results obtained in other centers [2-5] is presented in figures (c) and (d)

No energy or timing resolution data was available for the DMI system at Tokyo [3] and for the DMI-DR system at Southampton-Poole-Plymouth [5].

\section{Performance of other PET/CT systems}

Figures 6 and 7 present a comparison of previously published performance data for PET systems commercialized by Siemens Healthcare, Philips Medical Systems and GE Healthcare, with our findings.

Figure 6 shows the spatial resolutions at $1 \mathrm{~cm}[2,17,19-21,23,24,26]$, at $10 \mathrm{~cm}$ $[2,17,19-21,24,26]$, and $20 \mathrm{~cm}[2,17,20,24,26]$ radial offset in the (a) radial, (b) tangential/transverse, and (c) axial directions for the different systems. Figure $7 \mathrm{a}$ and b presents an appreciable comparison of the $\mathrm{CR}$ and BV results for the different systems. Count rate performances and scatter fraction for the different systems are presented in Fig. 7c. Finally, sensitivities at CFOV and at 10-cm-offset are presented in Fig. 7d.

\section{Discussion}

In this work, the physical performance of the GE Healthcare 4-ring DMI and DMI-DR systems at our Medical Center were measured using the NEMA guidelines for system evaluation. It is noteworthy that, except for their similar spatial resolution (mean relative deviation of $0.9 \%, 1.6 \%$, and $0.6 \%$ using FBP, VPHD (3D OSEM), VPHD-S (3D OSEM + PSF), respectively), performance of the 4-ring DMI system surpassed that of DMI-DR system. A similar spatial resolution between the two systems is expected due 
(a)

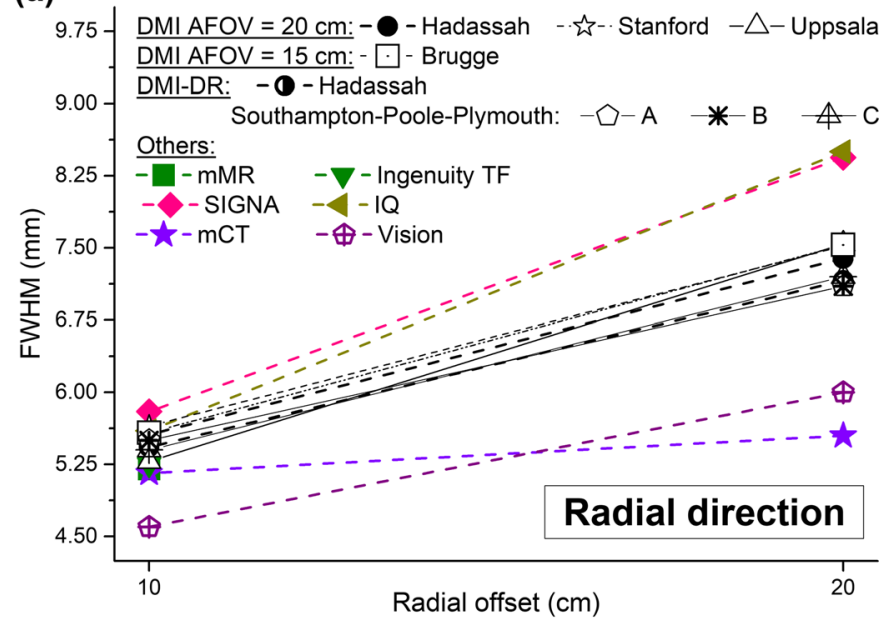

(b)

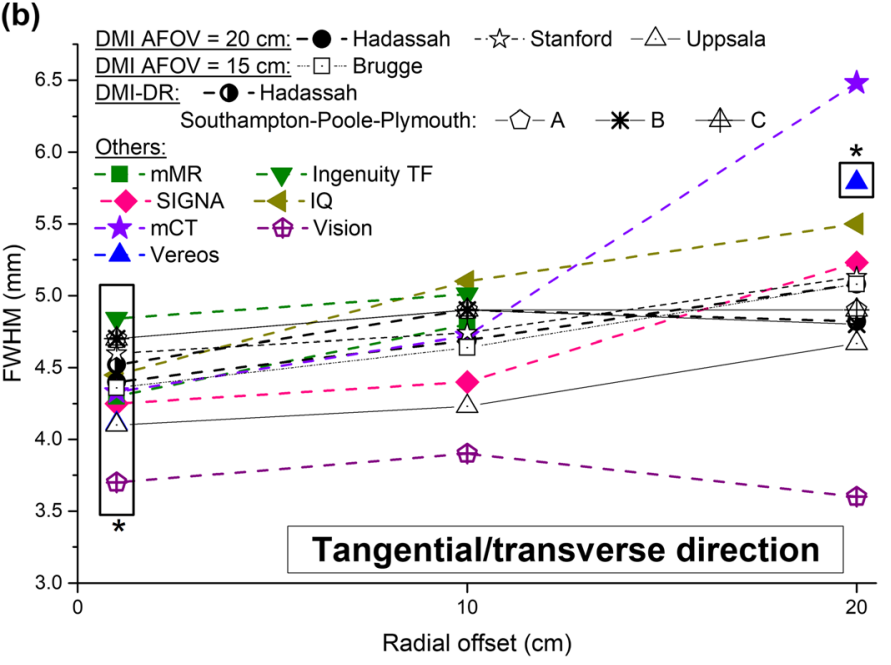

(c)

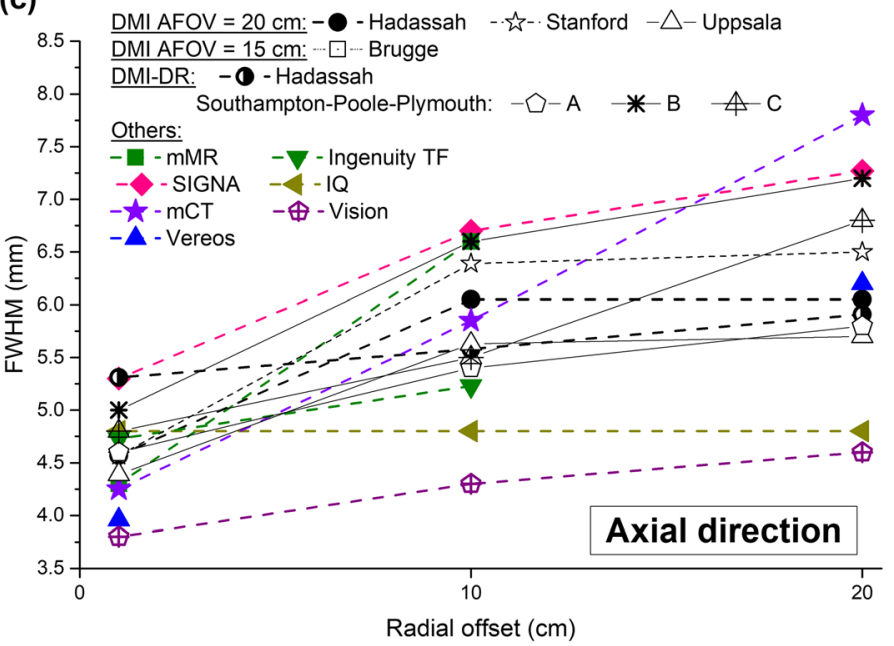

Fig. 6 (See legend on next page.) 
(See figure on previous page.)

Fig. 6 Resolution in mm (FWHM) for DMI, DMI-DR, Siemens Biograph mCT Flow [17, 18], Philips Ingenuity TF [21], Philips Vereos [22, 23], GE Discovery IQ [24, 25], GE SIGNA [26], Siemens Biograph mMR [18, 19] and digital Biograph Vision [20] systems in the a radial, $\mathbf{b}$ tangential or transverse (box with star) and $\mathbf{c}$ axial directions

to very similar LBS crystal dimensions and to the fact that crystal thickness is one of the main contributors to the intrinsic response function of the system.

TOF performance was better for the digital 4-ring DMI system, which showed a timing coincidence resolution of 377.3 ps compared with $552.7 \mathrm{ps}$ for the DMI-DR system. This result reflects the better timing performance of SiPM photodetectors compared with PMTs.

Sensitivity of the digital system in its $20 \mathrm{~cm}$ AFOV configuration $(13.25 \mathrm{cps} / \mathrm{kBq})$ was about twice that of the PMT-based PET/CT $(6.34 \mathrm{cps} / \mathrm{kBq})$, although a major contribution to the superior sensitivity of the DMI system is due to the difference in its extended AFOV (DMI-DR AFOV: $15.6 \mathrm{~cm}$ ). In principle, the doubled sensitivity of the DMI system should allow for the maintenance of SNR with reduced acquisition times or injected activity of the radiopharmaceutical (by a factor of two). Alternatively, the image SNR should be enhanced using the same acquisition time. True values and NECR peaks are $25 \%$ and 16\% higher for the 4-ring DMI system compared with the DMI-DR. At clinical concentrations, the differences in peak true values and NECR reach $46 \%$ and $37 \%$, respectively, in favor of the 4-ring DMI system. Figure $4 \mathrm{~b}$ shows that scatter fraction tends to increase with activity concentration, even though the

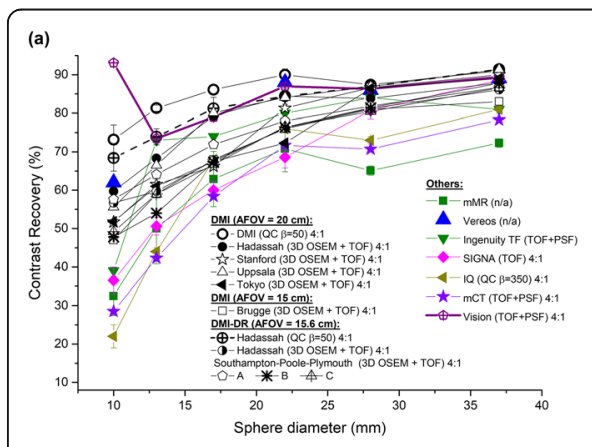

(c)

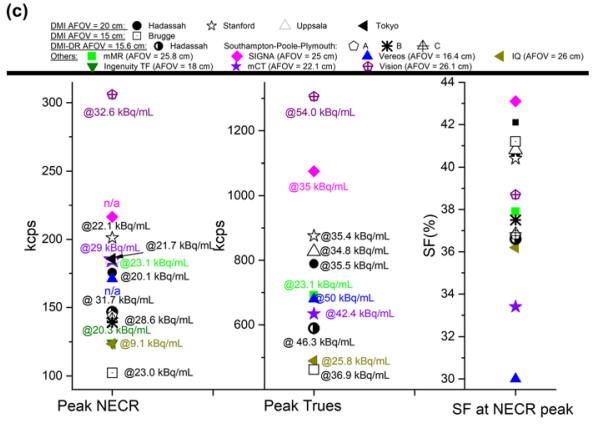

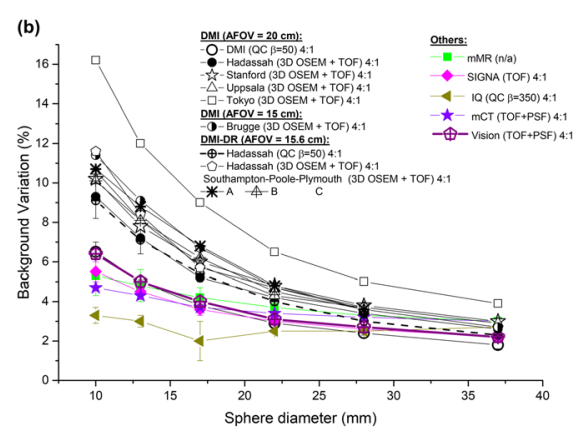

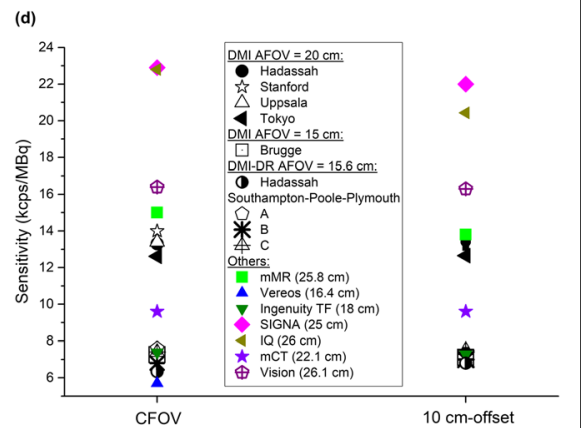

Fig. 7 a Contrast recovery (\%), b background variability (\%), c peak NECR, peak trues, scatter fraction at NECR peak, and corresponding activity concentrations and $\mathbf{d}$ sensitivity at CFOV and 10-cm radial offset for DMI, DMI-DR, Siemens Biograph mCT Flow [17, 18], Philips Ingenuity TF [21], Philips Vereos [22, 23], GE Discovery IQ [24, 25], GE SIGNA [26], Siemens Biograph mMR [18, 19] and digital Biograph Vision [20] 
attenuation/scatter distribution does not change, likely because of the pulse pile-up effect in the detectors. Indeed, if the detection of a $511 \mathrm{keV}$ photon is confounded with a scattered photon of energy less than the lower energy threshold, pile-up may lead to an energy signal that lies within the energy window. Therefore, the detection probability for this scattered photon and the measured scatter fraction increases as activity increases [27, 28].

Image quality was also better for 4-ring DMI for all reconstruction algorithms. The biggest difference, in contrast, was found when reconstructing images with the VPHD (3D OSEM) algorithm (12.5\%), while the difference decreased to its minimum (4.6\%) when using the $\mathrm{QC}$ algorithm with $\beta=50$. For background variability, the maximum difference is found for QC with $\beta=50$ (35.8\%) and the minimum for VPHD (1.5\%). Comparing the conventional 3D-OSEM (PSF+TOF) and Q.Clear algorithms revealed an improvement in PET image quality, with a higher coefficient of recovery and lower background variability using Q.Clear. Indeed, it has been previously shown that most Q.Clear reconstructions improve CR and BV compared with OSEM depending on the $\beta$-factor $[7,26]$. The increased CR with the Q.Clear reconstruction algorithm can be partly accounted for by the improved effective convergence of this algorithm. Also, the overall image quality using Q.Clear has been investigated and found to be the best reconstruction in most cases [26, 27], leading to a smooth and homogeneous appearance of background structures. Therefore, the use of Q.Clear can have implications for the assessment and quantification of treatment, especially in the detection of small foci. However, the optimal choice of $\beta$ in Q.Clear has been shown to be challenging [26]. Indeed, the $\beta$-value depends on many factors such as the isotope used, the injected dose, the acquisition time per bed position, etc. All these factors need to be considered and harmonized for an optimal integration of Q.Clear in clinical PET/CT imaging.

Figure 3 presents transverse views of the phantom and highlights Gibbs ringing artifacts for the different reconstructions. An overshoot of this artifact mainly influences the CR, with higher CRs for the $22 \mathrm{~mm}$ sphere than for the $28 \mathrm{~mm}$ sphere as observed in Fig. 2a for the different reconstruction algorithms and for the two systems. It is noteworthy that the influence of the Gibbs artifact seems to be more significant for the DMI system than for DMI-DR.

This study included a comparison between the 4-ring DMI performance results obtained in our institution with those from Stanford and Uppsala Universities [2] and Tokyo [3] for the same system and with Brugge [5] for the 3-ring DMI system. The results obtained in the present paper are in close agreement with these previous results, which is reassuring. For spatial resolution (Fig. 1), the present measurements show a similar behavior with a minimum mean relative deviation of $-1.17 \%$ for FBP from Brugge results and of $0.09 \%$ for VPHD (3D OSEM) from Tokyo. A maximum mean relative deviations of $-6.17 \% /-3.31 \%$ for $\mathrm{FBP} / \mathrm{VPHD}$ were obtained compared with Uppsala results. The mean deviation \pm SD over all the measurements is $-1.24 \pm 5.98 \%$. Image quality results for the 4-ring DMI system were also similar across all four centers (Hadassah, Uppsala, Stanford, Tokyo). Our findings show a minimum mean relative deviation of $-0.2 \%$ in CR from Uppsala and $6.4 \%$ in BV from Tokyo. Maximum mean relative deviations in $\mathrm{CR} / \mathrm{BV}$ of $-8.1 \% / 12.5 \%$ were found compared with Tokyo/Stanford. The 3-ring DMI system presented mean relative deviations of $-11.3 \% / 61 \%$ in CR/ BV from our results. Compared with the DMI-DR results, the 3-ring DMI showed, on 
average, lower CR by $3.6 \%$ and higher BV by $38.3 \%$. The peak NECR obtained in this work differed by only $5 \%$ from Uppsala and Tokyo and by $13 \%$ from Stanford. Compared with the 3-ring system, a much higher relative deviation of $42 \%$ is obtained. Compared with the DMI-DR system with a similar AFOV, the 3-ring DMI peak NECR is lower by $43 \%$. Around clinical activity concentrations, i.e., $5 \mathrm{kBq} / \mathrm{mL}$, NECR results obtained at Uppsala presented a relative deviation below 1\%. Scatter fraction results were also very similar for all activity concentrations across the Hadassah, Uppsala, and Stanford centers with less than $0.6 \%$ maximum deviation. From Brugge results, it is noteworthy that the scatter fraction for the DMI system in its shorter $15 \mathrm{~cm}$ AFOV configuration is similar or even higher to that of the 4-ring system. One might think that the scatter fraction observed at NECR peak for the 4-ring DMI was higher than DMI-DR (Fig. 4b) mainly due to its larger AFOV, keeping in mind that the energy acceptance windows are the same and energy resolutions are similar for the two systems. However, it is notable that for similar AFOV, the scatter fraction of the 3-ring DMI system is higher than the DMI-DR by about $5 \%$ in average. Sensitivity for our system at CFOV differed by $0.7 \%$ from Uppsala and by $5 \%$ from Stanford and Tokyo results. The CFOV sensitivity for the 3-ring DMI system obtained at Brugge was $45 \%$ lower than the 4-ring system and similar to that of the PMT-based DMI-DR system.

In view of the NEMA data of the 3-ring DMI $(15 \mathrm{~cm} \mathrm{AFOV})$ and of the DMI-DR $(15.6 \mathrm{~cm}$ AFOV) systems, it seems that the SiPMs LightBurst Digital Detectors do not present a real advantage compared with standard PMTs.

This work included also a comparison between four DMI-DR systems. Performance results obtained in the various sites differed from our findings by $2.8 \pm 11.4 \%$ in spatial resolution, $-2.4 \pm 5.8 \%$ in image quality, $-3.4 \pm 1.4 \%$ in peak NECR, and $15.3 \pm 5.4 \%$ in CFOV sensitivity. As specified by Tsu et al. [2], variations in the system manufacturing process may explain general trends in the variation between systems. Moreover, variations in activity calibrations used in the dose calibrator may also cause differences in performance results between the different systems.

Finally, the study presented a comparison of the DMI and DMI-DR system performance with that of other commercially available systems. We can note from Fig. 6 that spatial resolution results of the different PET systems commercialized by GE healthcare are very similar. However, the Siemens Biograph Vision seems to outperform all its competitors. The PET scanner with the narrowest crystal dimensions is the Vision system. Decreasing the crystal dimensions tends to improve the spatial resolution due to less light spread in the crystal. On the other hand, increasing the crystal thickness improves the sensitivity of the system. Indeed, for similar AFOV, the GE systems (DIQ and SIGNA) present thicker crystals and better sensitivity.

As specified for the DMI and DMI-DR systems, the TOF performance of the SiPMbased systems (Vereos, SIGNA, and Vision) is better than for the PMT-based PET/CT systems (Ingenuity $\mathrm{TF}, \mathrm{mCT}$ ). The Siemens mMR system lacks TOF capability due to the relatively low timing resolution of avalanche photodiodes (APDs). The GE DIQ uses BGO as the scintillating crystal and also lacks TOF capability.

Image quality results cannot be compared easily. Indeed, NEMA NU-2 2007 standards included longer acquisitions than the acquisition times specified in the NEMA NU-2 2012 standards, leading to favorable CR and BV in measurements using the earlier standards. Tests of the Biograph mMR PET/MR (Siemens Healthcare) [19] and the 
Ingenuity TF (Philips Medical Systems) [21] were performed according to the NEMA NU-2 2007 standards. Moreover, reconstruction algorithms are not the same for all the systems. From Fig. 7a and b, it is noteworthy that using the Q.Clear algorithm with $\beta=$ 50 gives the best results for the DMI and DMI-DR systems with with CR coefficients remaining at a constant high level $(>70 \%)$ across all sphere diameters. Results of the Siemens Biograph Vision are very close to those of DMI and DMI-DR (Q.Clear). The very high contrast recovery obtained in [20] for the smallest sphere is due to the Gibbs artifact when using PSF, as specified there. The influence of this artifact can also be observed for the $22 \mathrm{~mm}$ sphere in the Vision, DIQ, mCT and mMR systems on Fig. 7a. Moreover, it should be pointed out that although the 3-ring DMI gives intermediate $C R$ values, the $B V$ values are higher than its competitors.

The count rate results for the Biograph Vision system $(\mathrm{AFOV}=26.1 \mathrm{~cm})$ were the best of all PET/CT systems compared in this study (Fig. 7c), with NECR higher by $52 \%$ compared with the DMI (GE Healthcare) system (AFOV $=20 \mathrm{~cm}$ ) and by $145 \%$ compared with DIQ (GE Healthcare) system $(\mathrm{AFOV}=26 \mathrm{~cm}$ ) or the Ingenuity TF (AFOV $=18 \mathrm{~cm})$. The DMI NECR results were similar compared with the $\mathrm{mCT}(\mathrm{AFOV}=22.1$ $\mathrm{cm})$ and $\mathrm{mMR}(\mathrm{AFOV}=25.8 \mathrm{~cm})$ systems with a larger AFOV. For the DMI-DR system, $(\mathrm{AFOV}=15.6 \mathrm{~cm})$, peak NECR was higher by $15 \%$ compared with the DIQ or Ingenuity $\mathrm{TF}$ systems, but below other systems. The worse count rate results were obtained for the 3-ring DMI system, with NECR lower by about 200\% compared with Biograph Vision and by 67\% compared with Vereos (with a similar AFOV). We should note that the higher photon light output and faster decay time of LSO/LYSO crystals allow for higher count rates and result in a higher NECR value compared with BGO. However, the BGO detector does have better sensitivity than LYSO due to its better stopping power.

As shown in Fig. 7d, with the exception of the DIQ and Biograph Vision with their larger AFOV, the DMI system has the highest sensitivity compared with other PET/CT systems. The AFOV of the Biograph Vision $(26.1 \mathrm{~cm})$ is larger by about $30 \%$ compared with the DMI, leading to a similar level of sensitivity improvement. However, the DIQ, with a similar AFOV $(26 \mathrm{~cm})$, shows a sensitivity $74 \%$ higher than the DMI.

Sensitivity of the DMI-DR system is similar to that of the digital Philips Vereos PET/ CT system, which has a similar AFOV (DMI-DR: $15.6 \mathrm{~cm}$; Vereos: $16.4 \mathrm{~cm}$ ).

There are several limitations to our study. First, the NEMA NU 2-2012 analysis described in the present paper used the vendor-supplied software. As such, the work is not a completely independent assessment of performance. The development of homemade software for analysis would have added greater value to this paper. Also, the TOF performance of the scanners was not evaluated using the NEMA NU-2 2018 [29, 30] standards, where timing resolution has to be described as a function of the effective radioactivity concentration. Timing resolution is mainly degraded by pile-up, which varies with activity concentration.

\section{Conclusion}

According to measurements based on NEMA NU-2 2012 standards, the 4-ring digital DMI $(\mathrm{AFOV}=20 \mathrm{~cm})$ presents overall better performance compared with the PMT-based DMIDR $($ AFOV $=15.6 \mathrm{~cm})$ PET $/$ CT scanner, most notably with sensitivity approximately 
doubled. However, the Discovery MI system, in its $15 \mathrm{~cm}$ AFOV configuration, presents worse performance than the PMT-based system in terms of count rate, scatter fraction, and image quality. Improved image quality is obtained with the Q.Clear reconstruction algorithm, with increased CR and decreased BV. Finally, compared with other PMT-based PET/CT systems with similar or higher AFOV, the 4-ring DMI system with its LightBurst Digital Detectors offers better overall performance characteristics.

\section{Abbreviations}

ADC: Analog-to-digital converter; AFOV: Axial field-of-view; APDs: Avalanche photodiodes; ASIC: Application-specific integrated circuit; BV: Background variability; CFOV: Center of the field-of-view; CNR: Contrast-to-noise ratio; CR: Contrast recovery; DMI: Discovery MI; DMI-DR: Discovery MI-Digital Ready; FBP: Filtered back projection; FWHM: Full width half maximum; FWTM: Full width tenth maximum; GE: General Electric; IQBP: Image quality body phantom; LBS: Lutetium-based scintillators; LLD: Lower-level energy discriminators; NECR: Noise equivalent count rate; NEMA: National Electric Manufacturer's Association; OSEM: Ordered subsets expectation maximisation; PET/CT: Positron emission tomography/computed tomography; PMT: Photomultiplier tube; PSF: Point spread function; QC: Q.Clear; SD: Standard deviation; SiPM: Silicon photomultiplier; SNR: Signal-to-noise ratio; TDC: Time-to-digital converter; TFOV: Transaxial field-of-view; TOF: Time-of-flight; ULD: Upper-level energy discriminators; VPFX: VUE Point FX; VPHD: VUE Point HD

\section{Acknowledgements}

The authors would like to thank Dr. Eyal Rosenbach for helpful discussion and suggestions.

\section{Authors' contributions}

AC contributed to the design of this work. AC and RM were in charge of the preparation of the NEMA phantom and of the acquisition. $\mathrm{AC}$ analyzed the data. $\mathrm{AC}$ and $\mathrm{MO}$ wrote the manuscript. RM revised the manuscript. All authors read and approved the final manuscript.

\section{Funding}

Not applicable.

\section{Availability of data and materials}

NEMA raw data images are stored and archived on a CD at the Hadassah-Hebrew University Medical Center, Jerusalem, Israel.

\section{Ethics approval and consent to participate}

This article does not contain any studies with human participants or animals performed by any of the authors.

\section{Consent for publication}

Not applicable.

\section{Competing interests}

The authors declare that they have no conflict of interest.

Received: 16 May 2019 Accepted: 5 January 2020

Published online: 14 January 2020

\section{References}

1. National Electrical Manufacturers Association. Performance measurements of positron emission tomographs (PET). Rosslyn, USA: NEMA Standards Publication NU 2-2012; 2012.

2. Hsu DF, Ilan E, Peterson WT, Uribe J, Lubberink M, Levin CS. Studies of a next generation silicon-photomultiplier-based time-of-flight PET/CT System. J Nucl Med. 2017. https://doi.org/10.2967/jnumed.117.189514.

3. Wagatsuma K, Miwa K, Sakata M, Oda K, Ono H, Kameyama M, et al. Comparison between new-generation SiPM-based and conventional PMT-based TOF-PET/CT. Phys Med. 2017:42:203-10.

4. Vandendriessche D, Uribe J, Bertin H, De Geeter F. Performance characteristics of silicon photomultiplier based 15-cm AFOV TOF PET/CT. EJNMMI Phys. 2019;6:8.

5. Michopoulou S, O'Shaughnessy E, Thomson K, Guy MJ. Discovery molecular imaging digital ready PET/CT performance evaluation according to the NEMA NU2-2012 standard. Nucl Med Commun. 2019;40:270-7.

6. Discovery MI DR, Digital Ready, with LightBurst LBS Detector. GE Healthcare; 2017.

7. Hudson HM, Larkin RS. Accelerated image reconstruction using ordered subsets of projection data. IEEE Trans Med Imaging. 1994;13:601-9.

8. Tohme MS, Qi J. Iterative image reconstruction for positron emission tomography based on a detector response function estimated from point source measurements. Phys Med Biol. 2009;54:3709-25.

9. Steve Ross, Ph.D. Q.Clear [Internet]. GE Healthcare; Available from: http://www3.gehealthcare.com/ /media/documents/ us-global/products/pet-ct/whitepaper/q\%20clear/ge-healthcare-white-paper_qclear.pdf

10. Teoh EJ, McGowan DR, Macpherson RE, Bradley KM, Gleeson FV. Phantom and Clinical evaluation of the bayesian penalized likelihood reconstruction algorithm Q.Clear on an LYSO PET/CT System. J Nucl Med. 2015;56:1447-52. 
11. Q.Clear, 2x improvement in image quality, 2x improvement in PET quantification accuracy. GE Healthcare; 2015.

12. Discovery MI-NEMA test procedures and detector performance test. GE Healthcare; 2016.

13. Discovery MI DR-NEMA test procedures and detector performance test. GE Healthcare; 2016.

14. Bailey DL, Jones T, Spinks TJ. A method for measuring the absolute sensitivity of positron emission tomographic scanners. Eur J Nucl Med. 1991;18:374-9.

15. Jakoby BW, Bercier Y, Conti M, Casey ME, Bendriem B, Townsend DW. Physical and clinical performance of the $\mathrm{mCT}$ time-of-flight PET/CT scanner. Phys Med Biol. 2011;56:2375-89.

16. Uribe J, McDaniel DL, Stearns CW. Coincidence time correction (CTC) method for TOF-PET scanners with correction to account for misalignment of calibration phantom. 2013 IEEE Nucl Sci Symp Med Imaging Conf 2013 NSSMIC. 2013:1-3.

17. Rausch I, Cal-González J, Dapra D, Gallowitsch HJ, Lind P, Beyer T, et al. Performance evaluation of the biograph mCT Flow PET/CT system according to the NEMA NU2-2012 standard. EJNMMI Phys. 2015;2:26.

18. Karlberg AM, Sæther O, Eikenes L, Goa PE. Quantitative comparison of PET performance-Siemens Biograph $\mathrm{mCT}$ and $\mathrm{mMR}$. EJNMMI Phys [Internet]. 2016 [cited 2018 Feb 1];3. Available from: https://www.ncbi.n/m.nih.gov/pmc/articles/PMC4766138/

19. Delso G, Fürst S, Jakoby B, Ladebeck R, Ganter C, Nekolla SG, et al. Performance measurements of the Siemens mMR Integrated Whole-Body PETMR Scanner. J Nucl Med. 2011;52:1914-22.

20. van SJJ, de Jong J, Schaar J, Noordzij W, van Snick P, Dierckx R, et al. Performance characteristics of the digital Biograph Vision PET/CT system. J Nucl Med. 2019. https://doi.org/10.2967/jnumed.118.215418.

21. Kolthammer JA, Su K-H, Grover A, Narayanan M, Jordan DW, Muzic RF. Performance evaluation of the Ingenuity TF PET/ CT scanner with a focus on high count-rate conditions. Phys Med Biol. 2014;59:3843.

22. Zhang J, Miller M, Knopp M. SU-F-I-55: Performance evaluation of digital PET/CT: medical physics basis for the clinical applications. Med Phys. 2016;43:3399.

23. Miller M, Zhang J, Binzel K, Griesmer J, Laurence T, Narayanan M, et al. Characterization of the vereos digital photon counting PET system. J Nucl Med. 2015;56:434.

24. Reynés-Llompart G, Gámez-Cenzano C, Romero-Zayas I, Rodríguez-Bel L, Vercher-Conejero JL, Martí-Climent JM. Performance characteristics of the whole-body discovery IQ PET/CT system. J Nucl Med. 2017:58:1155-61.

25. Jha A, Mithun S, Singh A, Purandare N, Shah S, Agrawal A, et al. NEMA NU-2 2012 performance evaluation of Discovery IQ: a high sensitivity PET System. J Nucl Med. 2015;56:1847.

26. Grant AM, Deller TW, Khalighi MM, Maramraju SH, Delso G, Levin CS. NEMA NU 2-2012 performance studies for the SiPM-based ToF-PET component of the GE SIGNA PET/MR system. Med Phys. 2016;43:2334-43.

27. Stearns CW, Manjeshwar RM. Incorporating count-rate dependence into model-based PET scatter estimation. 2011 IEEE Nucl Sci Symp Conf Rec. 2011:3745-7.

28. Eriksson L, Watson CC, Wienhard K, Eriksson M, Casey ME, Knoess C, et al. The ECAT HRRT: an example of NEMA scatter estimation issues for LSO-based PET systems. IEEE Trans Nucl Sci. 2005;52:90-4.

29. National Electrical Manufacturers Association. Performance measurements of positron emission tomographs (PET). Rosslyn, USA: NEMA Standards Publication NU 2-2018; 2018

30. Zhang J, Maniawski P, Knopp MV. Performance evaluation of the next generation solid-state digital photon counting PET/CT system. EJNMMI Res. 2018;8:97

\section{Publisher's Note}

Springer Nature remains neutral with regard to jurisdictional claims in published maps and institutional affiliations.

\section{Submit your manuscript to a SpringerOpen ${ }^{\circ}$ journal and benefit from:}

- Convenient online submission

- Rigorous peer review

- Open access: articles freely available online

- High visibility within the field

- Retaining the copyright to your article

Submit your next manuscript at $\boldsymbol{\nabla}$ springeropen.com 